\title{
Consumo de sódio e síndrome metabólica: uma revisão sistemática
}

\author{
Sodium intake and metabolic syndrome: a systematic review
}

Flávio Sarno', Patricia Constante Jaime ${ }^{2}$, Sandra Roberta G. Ferreira'2 ${ }^{2}$ Carlos Augusto Monteiro²

1 Programa de Pós-graduação em Nutrição em Saúde Pública, Faculdade de Saúde Pública (FSP), Universidade de São Paulo (USP), São Paulo, SP, Brasil 2 Departamento de Nutrição, FSP/USP, São Paulo, SP, Brasil

\begin{abstract}
RESUMO
Estudos recentes mostram que restrições na ingestão de sódio podem aumentar a resistência à insulina (RI) e induzir alterações nas lipoproteínas séricas e em marcadores de inflamação semelhantes às encontradas na síndrome metabólica (SM). Realizou-se uma revisão sistemática da literatura sobre os efeitos da restrição do consumo de sódio sobre a SM ou a RI. Nove artigos foram incluídos na revisão. A restrição no consumo de sódio associou-se ao aumento da RI em dois artigos e a diminuição em três outros. Em sete dos nove artigos, a restrição na ingestão de sal determinou redução da pressão arterial e em dois artigos ocorreram efeitos adversos em marcadores da SM. A maioria dos estudos mostrou efeitos benéficos da restrição moderada de sódio da dieta associados ou não a outras modificações nutricionais ou ao aumento da atividade física. Novos estudos são necessários para avaliar os efeitos de reduções moderadas no consumo de sódio sobre a SM e a RI. Arq Bras Endocrinol Metab. 2009;53(5):608-16.

Descritores

Dieta hipossódica; sódio na dieta; síndrome metabólica; resistência à insulina; hipertensão
\end{abstract}

\begin{abstract}
Recent studies have shown that sodium intake restrictions may increase insulin resistance (IR) and induce changes on serum lipoproteins and on inflammation markers that are similar to those found in metabolic syndrome (MS). We performed a systematic review of literature regarding the effects of restricting sodium intake on MS or on IR. Nine articles were included in the review. Restriction of sodium consumption was associated with increase insulin resistance in two articles and with decrease in three others. In seven of nine articles, salt intake restriction determined blood pressure reduction, and in two articles adverse effects on markers of MS were found. Most studies showed beneficial effects of moderate sodium intake restriction, associated or not to others nutritional modifications or increased physical activity. Further studies are needed to evaluate the effects of moderate sodium consumption reductions on MS and IR. Arq Bras Endocrinol Metab. 2009;53(5):608-16.
\end{abstract}

Keywords

Sodium-restricted diet; dietary sodium; metabolic syndrome; insulin resistance, hypertension

\section{INTRODUÇÃO}

\section{Definição da síndrome metabólica e riscos}

$\mathrm{E}$ m 1988, Reaven descreveu a "Síndrome X", hoje Conhecida como síndrome metabólica (SM), como a ocorrência de resistência à insulina, hiperglicemia, elevação da lipoproteína de baixa densidade, diminuição da lipoproteína de alta densidade (HDL-c) e hipertensão arterial sistêmica (HAS) (1).
Apesar do debate sobre a existência como síndrome e da importância prognóstica da caracterização da mesma (2), organizações de saúde e sociedades científicas propuseram critérios diagnósticos para padronizar sua definição, tanto para fins clínicos como para pesquisas. Devido a diferenças étnicas no risco de desenvolvimento de certas doenças, alguns critérios e pontos de corte, especificamente para a circunferência da cintura, têm sido diferenciados de forma a adaptar a definição de SM às diversas populações (3). 
Em 1999, a World Health Organization (WHO) (4) e o European Group for the Study of Insulin Resistance (EGIR) (5) apresentaram as primeiras propostas, mas foi a do National Cholesterol Education Program (NCEP) (6) de 2001 a mais amplamente utilizada, em grande parte pela simplicidade dos parâmetros diagnósticos (glicemia de jejum $\geq 110 \mathrm{mg} / \mathrm{dL}$, pressão arterial $\geq 130 \times 85 \mathrm{mmHg}$, triglicérides - TG $\geq 150 \mathrm{mg} / \mathrm{dL}$, HDL-c $\leq 40 \mathrm{mg} / \mathrm{dl}$ ( 50 para mulheres) e circunferência abdominal $\geq 102 \mathrm{~cm}$ (88 para mulheres), sendo necessários pelo menos três desses fatores para se estabelecer o diagnóstico. Posteriormente, o NCEP sugeriu pequenas modificações em seus critérios, buscando incorporar o conhecimento atual na área (7).

Critérios mais abrangentes, incluindo determinações mais complexas, fizeram com que as definições da American Association of Clinical Endocrinologists e do American College of Endocrinology fossem menos empregadas na prática clínica e em pesquisas (8-10). Mais recentemente, a International Diabetes Federation (IDF) propôs um consenso para estabelecer os critérios diagnósticos da SM, que permite individualização para grupos populacionais (11). Essa proposta foi acatada pelo National Heart, Lung, and Blood Institute e American Heart Association (12), havendo, porém, diferenças nos pontos de corte de circunferência abdominal utilizados nas duas definições.

No Brasil, em 2005, foi publicada a primeira diretriz de diagnóstico e tratamento da SM (13) baseada nos critérios do NCEP (6). As sociedades científicas brasileiras recomendam considerar, além dos valores de corte, o uso de medicação anti-hipertensiva ou hipolipemiante para o estabelecimento da presença de HAS e dislipidemia e o diagnóstico prévio de diabetes mellitus (DM) para o preenchimento dos critérios dos respectivos distúrbios. Em face da recomendação da American Diabetes Association (14), o ponto de corte proposto para o diagnóstico de glicemia de jejum alterada passou de 110 para $100 \mathrm{mg} / \mathrm{dL}$. A recomendação brasileira estabelece que a circunferência abdominal seja medida a meia distância entre a crista ilíaca e o rebordo costal inferior e sugere que mulheres com circunferência abdominal entre 80 e $88 \mathrm{~cm}$ e homens entre 94 e $102 \mathrm{~cm}$ realizem monitorização mais frequente dos fatores de risco para doença cardiovascular (DCV).

Embora as definições disponíveis abordem alguns aspectos diferentes dentro do espectro de anormalidades da SM, a literatura é concordante em seus componentes essenciais: obesidade, hipertrigliceridemia, níveis baixos de HDL-c, HAS e intolerância à glicose. Além disso, tais definições compartilham do mesmo objetivo: auxiliar na identificação de indivíduos de risco para DCV e DM, que se beneficiariam com a intervenção precoce sobre essas anormalidades (3).

\section{Aspectos fisiopatológicos da síndrome metabólica}

A resistência à insulina, definida como redução na captação de glicose em resposta à ação insulínica, é apontada como a alteração central da SM. Os fatores de risco e os mecanismos associados à síndrome são múltiplos e não estão totalmente esclarecidos, envolvendo uma complexa inter-relação entre fatores genéticos e adquiridos (15-20). É provável que fatores agressores de diversas naturezas atinjam o organismo, desencadeando respostas compensatórias por meio do sistema neurohormonal, sendo a condição de resistência à insulina um dos principais marcadores desses processos (21).

Fatores ambientais relacionados ao estilo de vida têm sido fortemente implicados na fisiopatologia da SM. Esses últimos incluem hábitos dietéticos $(22)$ e inatividade física (23), ambos contribuindo para a obesidade e especialmente para o acúmulo de tecido adiposo na região abdominal, tecido diretamente envolvido na gênese da resistência à insulina. A grande liberação de ácidos graxos na circulação portal e sistêmica, decorrente da alta atividade lipolítica da gordura visceral, associada à produção de citocinas pró-inflamatórias e pró-aterogênicas, reduz a captação de glicose no fígado, musculatura esquelética e outros tecidos, gerando uma condição de alto risco cardiometabólico (24).

As anormalidades que compõem a SM se caracterizam por um alto grau de interação, uma contribuindo para o estabelecimento de outra. Porém, recentemente, dois estudos analisaram a sequência de eventos na fisiopatologia da SM. O primeiro, utilizando dados de três coortes, revelou que a circunferência da cintura basal pode predizer a deterioração de quatro a cinco outros componentes da SM, indicando que a obesidade visceral desempenha papel central no desenvolvimento da síndrome e parece preceder o aparecimento dos seus outros componentes (25). O segundo estudo, utilizando os dados do National Health and Nutrition Examination Survey (NHANES 1999 a 2002), sugeriu também a ocorrência de um efeito sequencial na instalação da SM: a partir da obesidade (identificada por meio do índice de massa corporal [IMC] ou pela circunferência da cintura), seguem-se a inflamação subclínica (definida pela proteína $\mathrm{C}$ reativa, PCR), a resistência à insulina e a dislipidemia (26). 


\section{Resistência à insulina, hipertensão arterial sistêmica e resposta ao sódio}

A captação tecidual de glicose mediada pela insulina varia amplamente entre os indivíduos e quanto mais insulino-resistente, maior a quantidade necessária de insulina a ser secretada no sentido de prevenir o desenvolvimento de diabetes melito tipo 2 . A presença de resistência à insulina e da hiperinsulinemia compensatória predispõe o indivíduo a vários distúrbios metabólicos e hemodinâmicos (27).

Modelos animais fornecem evidências da participação da resistência à insulina e do sódio na fisiopatogênese da HAS. Em ratos Dahl, modelos de pressão arterial sensível ao sal, demonstra-se que a resistência à insulina precede a elevação da pressão arterial e que a sobrecarga de sódio antecipa o surgimento da HAS (28).

Diferentes respostas pressóricas às variações na ingestão de sódio são encontradas em modelos animais de HAS e em seres humanos e as razões para tal heterogeneidade não estão completamente elucidadas. Também não está claro como a sensibilidade ao sal interfere no controle da pressão arterial de indivíduos com ou sem resistência à insulina. Apesar disso, reconhece-se que modificações na ingestão de sódio podem provocar efeitos no metabolismo da glicose.

Piora na sensibilidade à insulina foi obtida em modelos animais expostos a dietas hipossódicas. Ratos submetidos durante 12 semanas a uma dieta com baixos teores de sódio $(0,06 \%)$ apresentaram menor captação de glicose em comparação ao grupo controle, que consumiu dieta com níveis normais de sódio $(0,50 \%)$. Os resultados do estudo evidenciaram o envolvimento do aumento da atividade do sistema nervoso simpático e da diminuição da vasodilatação mediada pelo óxido nítrico no aumento da resistência à insulina induzida pela restrição de sal (29).

Já os achados em seres humanos não são consistentes: a restrição do consumo de sódio reduziu a resistência à insulina (30), não alterou (31) ou foi maior em subgrupos específicos de HAS (32).

A insulina promove a reabsorção renal de sódio e em condições de hiperinsulinemia espera-se exacerbação dessa ação. Em estados de resistência à insulina, esse efeito retentor está mantido nos rins, mostrando que a sensibilidade à insulina nas células tubulares proximais está preservada. De fato, comparando-se indivíduos com e sem SM, observou-se que os portadores da síndrome apresentavam reabsorção proximal de sódio significativamente maior (33).
Em suma, a resposta da pressão arterial diante do consumo de sódio é influenciada pela condição - possivelmente geneticamente determinada - de sensibilidade ao sal. A quantidade de sódio consumida pode alterar o metabolismo da glicose, enquanto as concentrações de insulina ou a sensibilidade tecidual a esse hormônio podem também interferir no controle renal do sódio corporal (34).

\section{Síndrome metabólica e consumo de sódio}

A excreção urinária do mineral - indicativa do seu consumo na dieta - mostra-se aumentada nos indivíduos com em comparação àqueles sem $\mathrm{SM}(4,0$ versus $3,4 \mathrm{~g} / \mathrm{d}$, $\mathrm{p}<0,01)(35)$. No estudo de Hoffmann e Cubeddu (36), a média de excreção de sódio urinário em homens e mulheres sem características da SM foi de 3,2 e $2,7 \mathrm{~g} / \mathrm{d}$, respectivamente, enquanto naqueles com quatro ou cinco componentes da síndrome foi de, respectivamente, 4,0 e $3,1 \mathrm{~g} / \mathrm{d}(\mathrm{p}<0,001)$, o que indicaria uma ingestão diária extra de 1,5 a $2 \mathrm{~g}$ de sal entre os indivíduos com a síndrome.

As mais recentes diretrizes para o tratamento da HAS na SM recomendam a redução no consumo de sódio $(11,12)$. A diretriz brasileira recomenda a redução da ingestão de sal de todas as formas, tanto para prevenção primária, como para tratamento não medicamentoso da síndrome (consumo de sal limitado a 6 g por dia) (13).

Porém, além das controvérsias a respeito do papel do consumo reduzido de sódio sobre o metabolismo da glicose, essa conduta poderia induzir alterações desfavoráveis nas lipoproteínas do plasma e em marcadores de inflamação, similares às encontradas na SM (37), efeitos indesejáveis para se minimizar o risco cardiovascular.

Resultados desfavoráveis à dieta hipossódica foram obtidos também em estudo de metanálise, quando se compararam dietas com baixos e altos teores de sódio: a primeira aumentou significativamente os níveis de renina, aldosterona, adrenalina, noradrenalina, colesterol total, lipoproteína de baixa densidade (LDL-c) e TG. Apesar das limitações decorrentes da inclusão de ensaios de curto prazo e do questionamento dos estudos que avaliaram eficácia ou efetividade, concluiu-se que a redução no consumo de sódio foi útil para diminuir a pressão arterial em indivíduos hipertensos no curto prazo e que a magnitude do efeito sobre a pressão arterial em indivíduos normotensos da raça branca não justifica uma recomendação generalizada para diminuir o consumo desse nutriente (38). 
Embora os estudos que avaliaram os efeitos de dietas restritas em sal em humanos tenham sido, em geral, realizados no estado de jejum e os resultados obtidos a partir de intervenções de curta duração, não se pode garantir que alterações metabólicas secundárias a essa restrição poderiam, eventualmente, superar o benefício esperado na aterogênese pela diminuição da pressão arterial.

O objetivo deste estudo foi realizar uma revisão da literatura no que se refere à associação entre $\mathrm{SM}$ ou resistência à insulina e consumo de sódio.

\section{METODOLOGIA}

Foram pesquisados artigos que analisaram os efeitos de restrições no consumo de sódio associados ou não a outras alterações nutricionais ou a outras intervenções na SM ou na resistência à insulina.

\section{Critérios de inclusão e exclusão de estudos}

Foram incluídos estudos originais, do tipo ensaio clínico (aleatorizados ou não, controlados ou não) e que apresentassem resultados referentes ao impacto de restrições no consumo de sódio na SM ou na resistência à insulina. Os demais critérios de inclusão foram: data da publicação do estudo entre janeiro de 2004 e dezembro de 2008 , terem sido realizados em adultos e publicados nos idiomas inglês e português. Foram excluídos os estudos observacionais e aqueles realizados em animais, recém-nascidos, crianças e adolescentes. Também não foram incluídos estudos que avaliaram o efeito de dietas com redução de sódio em associação ao uso de medicamentos.

\section{Estratégia de busca}

A pesquisa dos artigos foi realizada na base de dados PubMed, utilizando-se três conjuntos de intersecção de termos de busca bibliográfica: síndrome metabólica como desfecho (metabolic syndrome, cardiovascular syndrome, cardiometabolic syndrome, syndrome $X$, dysmetabolic syndrome, insulin resistance syndrome, insulin resistance, insulin sensitivity ou reaven syndrome); dieta como exposição (intake, consumption, diet, dietary ou excretion) e sódio como nutriente de interesse (salt, sodium ou $\mathrm{Na}$ ). Foram pesquisadas também as referências bibliográficas dos artigos incluídos.

Realizou-se uma primeira avaliação, tendo por base os títulos e o resumo dos artigos e rejeitaram-se aqueles que não preencheram os critérios de inclusão ou apre- sentaram algum dos critérios de exclusão. Quando um estudo não pode ser incluído ou rejeitado com certeza, o texto completo foi analisado secundariamente.

\section{Síntese e comparação dos estudos}

Foi realizada síntese narrativa dos estudos selecionados, apresentados segundo as características das populações e das intervenções. Número de participantes, idade média, IMC médio e prevalências de HAS e SM compuseram a caracterização das populações.

Para as intervenções, foram apresentados detalhes dos ensaios propriamente ditos, como tempo, tipo de intervenção (apenas dietética ou associada a recomendações de aumento da atividade física), níveis da restrição de sódio testados e forma de avaliação da adesão do participante em relação ao sódio consumido (coleta de sódio urinário de 24 horas ou aplicação de questionários - diários ou recordatórios alimentares de 24 horas).

Além disso, relacionaram-se os dados referentes à forma de avaliação do metabolismo da glicose (bomeostatic model assessment, HOMA, clamp euglicêmico hiperinsulinêmico, teste oral de tolerância à glicose, índice de sensibilidade à insulina ou glicemia) e da pressão arterial (médias de medidas de repouso ou de 24 horas), além da ocorrência de modificações de peso durante a restrição de sódio (aumento, diminuição ou sem modificações).

Sintetizou-se também a forma utilizada para comparar os resultados obtidos (diferenças intra-grupo $=$ apuradas no mesmo grupo de indivíduos antes e após o período de intervenção ou efeito líquido $=$ diferenças apuradas em grupos diferentes de indivíduos antes e após o período de intervenção) e resultados e conclusões principais.

Os desfechos analisados foram resistência à insulina, lípides plasmáticos, peso, cintura abdominal, pressão arterial, renina, aldosterona, atividade do sistema nervoso simpático e marcadores de inflamação. Foram consideradas significativas as diferenças apuradas cujos valores de $p$ foram menores que 0,05 .

Em decorrência da heterogeneidade das características das populações e das intervenções testadas, não foi possível realizar a síntese por metanálise.

\section{RESULTADOS}

A estratégia de pesquisa descrita encontrou 192 artigos, sendo 38 deles revisões. Ao final, nove artigos de oito estudos originais foram incluídos na revisão (dois 
artigos analisaram a mesma base de dados - estudo PREMIER) $(39,40)$.

\section{Populações}

O número de indivíduos estudados variou de 20 a 796, a idade média foi superior a 50 anos em cinco artigos, entre 40 e 50 anos em três e 30 anos em um artigo. O IMC médio dos participantes esteve acima do recomendado $\left(\geq 25 \mathrm{~kg} / \mathrm{m}^{2}\right)$ na maioria dos artigos. Todos os indivíduos apresentavam pré-hipertensão ou HAS em três artigos, em outros três a prevalência da doença variou de $12,3 \%$ a $65,2 \%$, em dois os participantes apresentavam pressão arterial normal e em um dos artigos a prevalência da doença não foi informada. Os critérios do NCEP 2001 foram utilizados para o diagnóstico de SM em quatro artigos, os da IDF 2005 em um deles, em três artigos a prevalência da síndrome não foi informada e em um deles os participantes eram indivíduos saudáveis. Quando relatada, a prevalência de SM variou de $26,8 \%$ a $100 \%$ dos participantes (Tabela 1 ).

\section{Intervenções}

A duração da intervenção variou de seis a sete dias em quatro artigos, três semanas em um deles e de três a seis meses em quatro artigos. Recomendações de aumento de atividade física, além de modificações no conteúdo de sódio da dieta, foram analisadas em dois artigos, sendo que quatro deles utilizaram o padrão dietético
DASH (Dietary Approaches to Stop Hypertension). Em todos os artigos, a restrição do consumo de sódio se situou abaixo de $2,4 \mathrm{~g} / \mathrm{d}$ (cinco deles na faixa entre 0,4 e $1,4 \mathrm{~g} / \mathrm{d}$ ) e a adesão ao consumo de sódio foi avaliada por meio da coleta de urina de 24 horas e/ou pela aplicação de questionários (diários ou recordatórios alimentares de 24 horas).

A maioria dos artigos utilizou o HOMA ou o clamp euglicêmico hiperinsulinêmico para analisar o metabolismo da glicose. Observou-se perda de peso dos participantes em quatro artigos e a pressão arterial foi avaliada pelas médias de duas ou três medidas de repouso na maioria dos artigos (Tabela 2).

\section{Efeitos da restrição de sódio sobre os desfechos analisados}

A restrição de sódio associou-se ao aumento da resistência à insulina em dois artigos $(37,41)$ e à diminuição em três outros $(39,40,42)$. Encontrou-se associação entre sensibilidade ao sal e resistência à insulina ou SM em três artigos (43-45). Foram observados redução da pressão arterial em sete artigos $(37,39,40,42-44,46)$, efeito benéfico nos lípides plasmáticos em três deles $(39,42,46)$ e diminuição da PCR e da atividade simpática em um artigo (42). Efeitos adversos, como aumento de TG, quilomícrons, marcadores de inflamação (PCR, interleucina 6 e fator de necrose tumoral alfa) foram mostrados em um artigo (37) e elevação de renina e aldosterona em dois artigos $(37,41)$ (Tabela 3$)$.

\begin{tabular}{|c|c|c|c|c|c|c|c|c|c|}
\hline $\begin{array}{l}\text { Autor, ano (referência) } \\
\text { Variáveis }\end{array}$ & $\begin{array}{l}\text { Nakandakare e } \\
\text { cols., } 2008 \\
\text { (37) }\end{array}$ & $\begin{array}{l}\text { Hoffmann } \\
\text { e Cubeddu, } \\
2007 \text { (43) }\end{array}$ & $\begin{array}{c}\text { Lien e } \\
\text { cols., 2007 } \\
\text { (39) }\end{array}$ & $\begin{array}{l}\text { Townsend } \\
\text { e cols., } \\
2007 \text { (41) }\end{array}$ & $\begin{array}{l}\text { Uzu e } \\
\text { cols., } 2006 \\
\text { (44) }\end{array}$ & $\begin{array}{l}\text { Straznicky } \\
\text { e cols., } \\
2005 \text { (42) }\end{array}$ & $\begin{array}{l}\text { Azadbakht } \\
\text { e cols., } \\
2005 \text { (46) }\end{array}$ & $\begin{array}{l}\text { Ard e } \\
\text { cols., } 2004 \\
\text { (40) }\end{array}$ & $\begin{array}{l}\text { Vedovato e } \\
\text { cols., } 2004 \\
\text { (45) }\end{array}$ \\
\hline $\begin{array}{l}\text { Participantes } \\
\text { (Homens/Mulheres) }\end{array}$ & $\begin{array}{c}41 \\
(15 / 26)\end{array}$ & $\begin{array}{c}301 \\
(87 / 214)\end{array}$ & $\begin{array}{c}796 \\
(304 / 492)\end{array}$ & $\begin{array}{c}20 \\
(12 / 8)\end{array}$ & $\begin{array}{c}56 \\
(35 / 21)\end{array}$ & $\begin{array}{c}23 \\
(15 / 8)\end{array}$ & $\begin{array}{c}116 \\
(34 / 82)\end{array}$ & $\begin{array}{l}52 \\
(16 / 36)\end{array}$ & $\begin{array}{l}41^{*} \\
(31 / 10)\end{array}$ \\
\hline Média de idade (anos) & 53,5 & 41,5 & $\begin{array}{l}49,9 \text { sem } \\
\text { SM } \\
49,7 \text { com } \\
\text { SM }\end{array}$ & 30 & $\begin{array}{l}51,5 \text { sem } \\
\text { SM } \\
50,2 \text { com } \\
\text { SM }\end{array}$ & 58,0 & 41,2 & 51,7 & $57-60$ \\
\hline Média do IMC (kg/m²) & 26,1 & $\begin{array}{l}27,9 \text { sem } \\
\text { SM } \\
32,2 \text { com } \\
\text { SM }\end{array}$ & NR & 23,1 & $\begin{array}{l}23,1 \text { sem } \\
\text { SM } \\
26,6 \text { com } \\
\text { SM }\end{array}$ & 33,3 & 29,7 & 32,6 & $28-29$ \\
\hline Prevalência de HAS & $100 \%$ & $12,3 \%$ & $100 \%$ & $0 \%$ & $100 \%$ & $65,2 \%$ & NR & $36,5 \%$ & $0 \%$ \\
\hline Prevalência de SM & NR & $\begin{array}{c}\text { NCEP-01 } \\
34 \%\end{array}$ & $\begin{array}{c}\text { NCEP-01 } \\
50,1 \%\end{array}$ & $0 \%$ & $\begin{array}{l}\text { IDF-05 } \\
26,8 \%\end{array}$ & $\begin{array}{c}\text { NCEP-01 } \\
100 \%\end{array}$ & $\begin{array}{c}\text { NCEP-01 } \\
100 \%\end{array}$ & NR & NR \\
\hline
\end{tabular}

NR: não relatado; IMC: índice de massa corporal; HAS: pré ou hipertensão arterial sistêmica; SM: síndrome metabólica - critério diagnóstico; NCEP-01: National Cholesterol Education Program 2001; IDF-05: International Diabetes Federation 2005.

* Participantes diabéticos. 


\begin{tabular}{|c|c|c|c|c|c|c|c|c|c|}
\hline $\begin{array}{l}\text { Autor, ano (referência) } \\
\text { Variáveis }\end{array}$ & $\begin{array}{l}\text { Nakandakare } \\
\text { e cols., } 2008 \\
\text { (37) }\end{array}$ & $\begin{array}{l}\text { Hoffmann } \\
\text { e Cubeddu, } \\
2007 \text { (43) }\end{array}$ & $\begin{array}{l}\text { Lien e } \\
\text { cols., } 2007 \\
\text { (39) }\end{array}$ & $\begin{array}{l}\text { Townsend } \\
\text { e cols., } \\
2007 \text { (41) }\end{array}$ & $\begin{array}{c}\text { Uzu e } \\
\text { cols., } 2006 \\
\text { (44) }\end{array}$ & $\begin{array}{l}\text { Straznicky } \\
\text { e cols., } \\
2005 \text { (42) }\end{array}$ & $\begin{array}{l}\text { Azadbakht } \\
\text { e cols., } \\
2005 \text { (46) }\end{array}$ & $\begin{array}{l}\text { Ard e } \\
\text { cols., 2004 } \\
\text { (40) }\end{array}$ & $\begin{array}{l}\text { Vedovato e } \\
\text { cols., } 2004 \\
\text { (45) }\end{array}$ \\
\hline Duração & 3 semanas & 1 semana & 6 meses & 6 dias & 1 semana & 3 meses & 6 meses & 6 meses & 1 semana \\
\hline Intervenção & Dieta & Dieta & $\begin{array}{l}\text { Dieta + } \\
\text { atividade } \\
\text { física }\end{array}$ & Dieta & Dieta & Dieta & Dieta & $\begin{array}{l}\text { Dieta + } \\
\text { atividade } \\
\text { física }\end{array}$ & Dieta \\
\hline Restrição de sódio (g/d) & 1,40 & 1,00 & 2,30 & 0,46 & $0,40-1,20$ & 2,30 & 2,40 & 2,40 & 0,60 \\
\hline Avaliação do sódio da dieta & $\mathrm{NaU}$ & $\mathrm{NaU}$ & $\mathrm{NaU}$ & $\mathrm{NaU}$ & $\mathrm{NaU}$ & $\mathrm{NaU}$ & $Q$ & $Q$ & $\mathrm{NaU}$ \\
\hline Metabolismo da glicose & HOMA & TOTG & HOMA & Clamp & HOMA & HOMA & Glicemia & ISS & Clamp* \\
\hline Modificação do peso & Não & NR & Redução & Não & NR & Redução & Redução & Redução & NR \\
\hline Pressão arterial & Média de 24 h & $\begin{array}{l}\text { Média em } \\
\text { repouso }\end{array}$ & $\begin{array}{l}\text { Média em } \\
\text { repouso }\end{array}$ & $\begin{array}{l}\text { Média em } \\
\text { repouso }\end{array}$ & $\begin{array}{c}\text { Média de } 24 \\
\text { h }\end{array}$ & $\begin{array}{l}\text { Média em } \\
\text { repouso }\end{array}$ & $\begin{array}{l}\text { Média em } \\
\text { repouso }\end{array}$ & $\begin{array}{l}\text { Média em } \\
\text { repouso }\end{array}$ & $\begin{array}{c}\text { Média de } 24 \\
\text { h }\end{array}$ \\
\hline
\end{tabular}

NR: não relatado; NaU: sódio urinário de 24 horas; Q: questionário (diário ou recordatório alimentar 24 h); HOMA: Homeostatic model assessment; TOTG: teste oral de tolerância à glicose; ISS: índice de sensibilidade à insulina.

*0 clamp euglicêmico hiperinsulinêmico foi realizado em 18 participantes.

\section{DISCUSSÃO}

A maioria dos estudos analisados mostrou efeitos benéficos da restrição moderada de sódio da dieta, associada ou não a outras modificações nutricionais ou ao aumento da atividade física, tanto em parâmetros metabólicos quanto na pressão arterial.

Os artigos que constataram efeitos adversos frente a uma ingestão reduzida de sódio utilizaram reduções além da usualmente recomendada de $2,4 \mathrm{~g} / \mathrm{d}$ para prevenção e tratamento da HAS na SM (13). Quando a redução do sódio na dieta foi moderada, observou-se diminuição das concentrações séricas de PCR, efeito desejável em termos de risco cardiovascular (42). Deve-se salientar, entretanto, que a constatação de efeitos adversos ou benéficos esteve limitada somente aos desfechos analisados e apenas dois artigos estudaram os efeitos da redução de sódio sobre marcadores de inflamação $(37,42)$, dois sobre renina e aldosterona $(37,41)$ e um sobre a atividade simpática (42).

$\mathrm{O}$ efeito da restrição no consumo de sódio sobre a resistência à insulina parece depender do nível da restrição planejada e, possivelmente, da duração da intervenção. Em estudo de Gomi e cols., a restrição de sódio para $2,3 \mathrm{~g} / \mathrm{d}$ por uma semana não resultou em mudanças significativas da sensibilidade à insulina e trouxe diminuições significativas da pressão arterial, ao passo que reduções maiores do consumo de sódio (para $0,7 \mathrm{~g} / \mathrm{d}$ ) por sete dias afetaram adversamente $\mathrm{o}$ metabolismo da glicose, sem ganhos maiores na pressão arterial, sugerindo que o consumo moderado de sódio pode diminuir a pressão arterial sem modificar a sensibilidade à insulina em indivíduos com HAS (47).
Apesar de, em parte dos artigos apresentados, apenas os efeitos de curto prazo terem sido analisados, estudo de metanálise mostrou que a redução moderada de consumo de sal por um período de quatro ou mais semanas apresentou efeito significativo na diminuição da pressão arterial em indivíduos com pressão arterial normal ou elevada (48).

Diferentemente da suplementação ou restrição de nutrientes específicos, a utilização de dietas completas nos estudos de intervenção não permite a determinação de quais de seus componentes individuais foram os responsáveis pelas modificações metabólicas observadas ou se essas modificações se deveram ao conjunto dos vários componentes. Quatro estudos testaram a dieta DASH, que apresenta maior conteúdo de cálcio, potássio, magnésio, nozes, grãos integrais e menor conteúdo de carne vermelha, gordura total, gordura saturada, colesterol e doces, quando comparada com uma dieta padrão voltada para redução de peso (49). O consumo de grandes quantidades de laticínios, típico do padrão dietético DASH, associa-se inversamente à $S M$ e à resistência à insulina e a restrição de sódio e os altos níveis de potássio, também presentes neste padrão de dieta, apresentam efeitos favoráveis sobre a pressão arterial. O benefício da dieta DASH no metabolismo da glicose pode ser resultado do alto consumo de fibras ou de grãos integrais, alimentos com baixo índice glicêmico (50).

Mesmo com a utilização de análise e controle estatístico de variáveis importantes para os desfechos, como peso ou nível de atividade física, como forma de se obterem os efeitos líquidos das intervenções, não se pode afastar a possibilidade de que os resultados observados 
Tabela 3. Artigos incluídos na revisão de acordo com autor, ano de publicação e características das intervenções, diferenças avaliadas e resultados e conclusões principais

\begin{tabular}{|c|c|c|c|c|}
\hline $\begin{array}{l}\text { Intervenção } \\
\text { Autor, ano, } \\
\text { (referência) }\end{array}$ & $\begin{array}{l}\text { Características das dietas } \\
\text { e das intervenções }\end{array}$ & $\begin{array}{l}\text { Diferenças } \\
\text { avaliadas }\end{array}$ & Resultados principais & Conclusões principais \\
\hline $\begin{array}{l}\text { Nakandakare e cols., } \\
2008 \text { (37) }\end{array}$ & $\begin{aligned} \text { A- sódio } & =3,7 \mathrm{~g} / \mathrm{d} \text { e B- sódio } \\
& =1,4 \mathrm{~g} / \mathrm{d}\end{aligned}$ & Intragrupo & $\begin{array}{l}\uparrow \text { TG, quilomícrom-colesterol, PCR, } \\
\text { TNF-alfa, IL-6, atividade da renina, } \\
\text { aldosterona, insulina e HOMA } \\
\downarrow \downarrow \text { PAS, PAD, PAM }\end{array}$ & $\begin{array}{l}\text { A restrição no consumo de sódio } \\
\text { induziu alterações nas lipoproteínas } \\
\text { séricas e marcadores de inflamação } \\
\text { características da SM }\end{array}$ \\
\hline $\begin{array}{l}\text { Hoffmann e Cubeddu, } \\
2007 \text { (43) }\end{array}$ & $\begin{array}{c}\text { A- sódio = 3,2 g/d, B- sódio = } \\
7,2 \mathrm{~g} / \mathrm{d} \mathrm{e} \mathrm{C-}=1,0 \mathrm{~g} / \mathrm{d}\end{array}$ & Efeito líquido & $\begin{array}{c}\text { Maior } \downarrow \text { PAS e PAD na SM } \\
\downarrow \text { PAS e PAD proporcional ao número } \\
\text { de características de SM } \\
\downarrow \text { prevalência de HAS na SM } \\
(23,8 \text { para } 8,2 \%)\end{array}$ & $\begin{array}{l}\text { Aumento da prevalência da } \\
\text { sensibilidade ao sal no grupo com } \\
\text { SM, estando esta diretamente } \\
\text { relacionada ao número de } \\
\text { características da síndrome em cada } \\
\text { indivíduo }\end{array}$ \\
\hline Lien e cols., 2007 (39) & $\begin{array}{l}\text { A- aconselhamento individual, } \\
\text { B- intervenção comportamental } \\
\text { intensa e C- = B + dieta DASH } \\
\text { Em B e C- sódio } \leq 2,3 \mathrm{~g} / \mathrm{d}\end{array}$ & Efeito líquido & $\begin{array}{c}\downarrow \text { PAS }(\mathrm{mmHg}) \text { sem e com SM: } \\
\text { A: }-6,2 \text { e }-6,8(p=0,60) ; B:-12,0 \text { e } \\
-8,4(p=0,00) ; C:-11,2 \text { e }-9,9 \\
(p=0,23) \\
\downarrow \text { HOMA, colesterol total em B e C } \\
\text { (com SM) }\end{array}$ & $\begin{array}{c}\text { A SM atenuou a diminuição da PAS } \\
\text { em B, atenuação superada com a dieta } \\
\text { DASH em C }\end{array}$ \\
\hline $\begin{array}{l}\text { Townsend e cols., } 2007 \\
\text { (41) }\end{array}$ & $\begin{aligned} \text { A- sódio }= & 4,6 \mathrm{~g} / \mathrm{d} \text { e B- sódio }= \\
& 0,46 \mathrm{~g} / \mathrm{d}\end{aligned}$ & Intragrupo & $\begin{array}{c}\downarrow \text { captacão de glicose mediada pela } \\
\text { insulina }\left(7,41 \text { versus } 6,1 \mathrm{mg} / \mathrm{kg}^{\star} \mathrm{min},\right. \\
\quad p=0,03) \rightarrow \mathrm{PA} \\
\uparrow \text { de renina e aldosterona }\end{array}$ & $\begin{array}{l}\text { A restrição de sal reduziu a } \\
\text { sensibilidade à insulina }\end{array}$ \\
\hline Uzu e cols., 2006 (44) & $\begin{aligned} \text { A- sódio = } & 4 \text { a 4,8 g/d e B- sódio = } \\
& \text { 0,4 a 1,2 g/d }\end{aligned}$ & Efeito líquido & $\begin{array}{c}\uparrow \text { prevalência de HAS sódio sensível } \\
\text { no grupo com SM }(70,6 \text { versus } \\
36,0 \%, p=0,02) \\
0 \text { efeito de B na queda da PAM } \\
\text { noturna foi maior nos pacientes com } \\
\text { SM }\end{array}$ & $\begin{array}{c}\text { Foi mostrada uma relação significativa } \\
\text { entre SM, sensibilidade da PA ao } \\
\text { sódio e diminuição da PA noturna }\end{array}$ \\
\hline $\begin{array}{l}\text { Straznicky e cols., } 2005 \\
\text { (42) }\end{array}$ & $\begin{array}{l}\text { Dieta DASH modificada (sódio = } \\
\text { 2,3 g/d) e redução de energia para } \\
\text { diminuição de peso }\end{array}$ & Intragrupo & $\begin{array}{c}\downarrow \text { PAS, peso, IMC, CA, colesterol } \\
\text { total, TG, glicemia, insulina, PCR, } \\
\text { atividade simpática e HOMA } \\
\uparrow \mathrm{HDL}\end{array}$ & $\begin{array}{c}\text { A diminuição de peso por meio de } \\
\text { uma dieta hipocalórica com restrição } \\
\text { moderada de sódio trouxe melhorias } \\
\text { nos componentes da SM }\end{array}$ \\
\hline $\begin{array}{l}\text { Azadbakht e cols., } 2005 \\
\text { (46) }\end{array}$ & $\begin{array}{c}\text { A- dieta controle, B- }=\text { A e } \\
\downarrow 500 \mathrm{kcal} / \mathrm{d} \text { para redução de peso } \\
\text { e C- dieta DASH }(\downarrow 500 \mathrm{kcal} / \mathrm{d} \\
\text { e sódio }<2,4 \mathrm{~g} / \mathrm{d})\end{array}$ & Efeito líquido & $\begin{array}{c}C \text { versus } B \text { : > aumento de } H D L \text { e > } \\
\text { redução de } P A S, P A D, T G, \text { glicemia, } \\
\text { peso e prevalência SM }\end{array}$ & $\begin{array}{l}\text { A dieta DASH diminuiu a maioria dos } \\
\text { fatores de risco metabólicos }\end{array}$ \\
\hline Ard e cols., 2004 (40) & $\begin{array}{c}\text { A- aconselhamento individual, B- } \\
\text { Intervenção comportamental intensa e } \\
\text { C- = B + dieta DASH. Em B e } \\
\text { C- sódio } \leq 2,4 \mathrm{~g} / \mathrm{d}\end{array}$ & Efeito líquido & $\begin{array}{c}\text { B - A: } \downarrow \text { peso, IMC, insulina, glicemia } \\
C-A: \uparrow \text { ISS (1,96 para 2,95 - } \\
p=0,047) \text { e } \downarrow \text { PAS, peso, CA, IMC } \\
C-B: \rightarrow \text { ISS ( } p=0,62) \text {, > razão de } \\
\text { mudança insulina-glicose }\end{array}$ & $\begin{array}{l}\text { A dieta DASH aumentou a ação da } \\
\text { insulina além do efeito de B }\end{array}$ \\
\hline $\begin{array}{l}\text { Vedovato e cols., } 2004 \\
(45)\end{array}$ & A- sódio = 0,6 g/d e B- sódio $6 \mathrm{~g} / \mathrm{d}$ & Análise de correlação & $\begin{array}{l}\text { As mudanças na PAM induzidas pelo } \\
\text { sal estiveram inversamente } \\
\text { relacionadas com a sensibilidade à } \\
\text { insulina ( } r=-0,51, p=0,01)\end{array}$ & $\begin{array}{c}\text { A resistência à insulina pode } \\
\text { contribuir para a maior sensibilidade } \\
\text { ao sal }\end{array}$ \\
\hline
\end{tabular}

$1 \mathrm{~g}$ sal $\approx 0,4 \mathrm{~g}$ de sódio $\approx 17,4 \mathrm{mmol}$ de sódio; $\uparrow:$ aumento, $\downarrow$ : redução, $\rightarrow$ : sem modificação, PA: pressão arterial; PAS: pressão arterial sistólica; PAD: pressão arterial diastólica; PAM: pressão arterial média; TG: triglicérides; LDL: lipoproteína de baixa densidade; HDL: lipoproteína de alta densidade; PCR: proteína C reativa; TNF-alfa: fator de necrose tumoral alfa; IL-6: interleucina 6; HOMA: homeostasis model assessment, SM: síndrome metabólica; ISS: índice de sensibilidade à insulina; IMC: índice de massa corporal; CA: circunferência abdominal.

$\mathrm{A}, \mathrm{B}$ e C referem-se às características das dietas utilizadas nas intervenções e os resultados principais, quando não especificados, referem-se àquelas com restrição de sódio.

nos estudos em que ocorreram modificações nessas variáveis possam ter sido, em parte, influenciados pelos benefícios obtidos tanto pela perda de peso como pelo aumento do preparo físico dos participantes $(23,51)$.
A sensibilidade à insulina foi medida, na maioria dos estudos, por meio do HOMA, fórmula matemática que estima a captação de glicose em jejum; porém, a correlação desse índice com o clamp euglicêmico, padrão- 
ouro para avaliação da sensibilidade à insulina, é bastante satisfatória $(52)$.

O número limitado de artigos revisados e diferenças relativas às populações e ao conteúdo das intervenções dificultaram a generalização dos resultados obtidos e recomendam a realização de mais estudos sobre os efeitos de reduções moderadas no consumo de sódio sobre a SM e resistência à insulina.

Declaração: os autores declaram não haver conflitos de interesse científico neste estudo.

\section{REFERÊNCIAS}

1. Reaven GM. Banting lecture 1988. Role of insulin resistance in human disease. Diabetes. 1988;37(12):1595-607.

2. Kahn R, Buse J, Ferrannini E, Stern M, American Diabetes A, European Association for the Study of $D$. The metabolic syndrome: time for a critical appraisal: joint statement from the American Diabetes Association and the European Association for the Study of Diabetes. Diabetes Care. 2005;28(9):2289-304.

3. Levesque J, Lamarche B. The metabolic syndrome: definitions, prevalence and management. J Nutrigenet Nutrigenomics. 2008;1:100-8.

4. WHO. World Health Organization. Definition, Diagnosis and Classification of Diabetes Mellitus and its Complications. Report of a WHO consultation: WHO/NCD/NCS1999 Contract 99.2.

5. Balkau B, Charles MA. Comment on the provisional report from the WHO consultation. European Group for the Study of Insulin Resistance (EGIR). Diabet Med. 1999;16(5):442-3.

6. III ATP. Executive summary of the third report of the National Cholesterol Education Program (NCEP) Expert Panel on Detection, Evaluation, and Treatment of High Blood Cholesterol in Adults. JAMA. 2001;285(19):2486-97.

7. Grundy SM, Brewer HB Jr, Cleeman JI, Smith SC Jr, Lenfant C, American Heart A, et al. Definition of metabolic syndrome: report of the National Heart, Lung, and Blood Institute/American Heart Association conference on scientific issues related to definition. Circulation. 2004;109(3):433-8.

8. Bloomgarden ZT. American Association of Clinical Endocrinologists (AACE) consensus conference on the insulin resistance syndrome: 25-26 August 2002, Washington, DC. Diabetes Care. 2003;26(3):933-9.

9. Bloomgarden ZT. American Association of Clinical Endocrinologists (AACE) consensus conference on the insulin resistance syndrome: 25-26 August 2002, Washington, DC. Diabetes Care. 2003;26(4):1297-303.

10. Einhorn D, Reaven GM, Cobin RH, Ford E, Ganda OP, Handelsman $\mathrm{Y}$, et al. American College of Endocrinology position statement on the insulin resistance syndrome. Endocr Pract. 2003;9(3):237-52.

11. Alberti KG, Zimmet P, Shaw J. Metabolic syndrome: a new worldwide definition. A Consensus Statement from the International Diabetes Federation. Diabet Med. 2006;23(5):469-80.

12. Grundy SM, Cleeman JI, Daniels SR, Donato KA, Eckel RH, Franklin $\mathrm{BA}$, et al. Diagnosis and management of the metabolic syndrome: an American Heart Association/National Heart, Lung, and Blood Institute Scientific Statement. Circulation. 2005;112(17):2735-52.

13. Sociedade Brasileira de $H$, Sociedade Brasileira de $C$, Sociedade Brasileira de Endocrinologia e M, Sociedade Brasileira de D, Sociedade Brasileira de Estudos da O. [l Brazilian guidelines on diagnosis and treatment of metabolic syndrome]. Arq Bras Cardiol. 2005;84 Suppl 1:1-28.
14. American Diabetes A. Diagnosis and classification of diabetes mellitus. Diabetes Care. 2004;27 Suppl 1:S5-10.

15. Gallagher EJ, LeRoith D, Karnieli E. The metabolic syndrome from insulin resistance to obesity and diabetes. Endocrinol Metab Clin North Am. 2008;37(3):559-79, vii.

16. Kressel G, Trunz B, Bub A, Hulsmann O, Wolters M, Lichtinghagen $\mathrm{R}$, et al. Systemic and vascular markers of inflammation in relation to metabolic syndrome and insulin resistance in adults with elevated atherosclerosis risk. Atherosclerosis. 2009;202(1):263-71.

17. Goossens GH. The role of adipose tissue dysfunction in the pathogenesis of obesity-related insulin resistance. Physiol Behav. 2008;94(2):206-18.

18. Lara-Castro C, Garvey WT. Intracellular lipid accumulation in liver and muscle and the insulin resistance syndrome. Endocrinol Metab Clin North Am. 2008;37(4):841-56.

19. Grattagliano I, Palmieri VO, Portincasa P, Moschetta A, Palasciano G. Oxidative stress-induced risk factors associated with the metabolic syndrome: a unifying hypothesis. J Nutr Biochem. 2008;19(8):491-504.

20. Briana $D$, Malamitsi-Puchner A. Intrauterine growth restriction and adult disease: the role of adipocytokines. Eur $\mathrm{J}$ Endocrinol. 2009;160(3):337-47.

21. Sjostrand M, Eriksson JW. Neuroendocrine mechanisms in insulin resistance. Mol Cell Endocrinol. 2009;297(1-2):104-11.

22. Esposito K, Ceriello A, Giugliano D. Diet and the metabolic syndrome. Metab Syndr Relat Disord. 2007;5(4):291-6.

23. Lakka TA, Laaksonen DE. Physical activity in prevention and treatment of the metabolic syndrome. Appl Physiol Nutr Metab. 2007;32(1):76-88.

24. Despres JP, Lemieux I, Bergeron J, Pibarot P, Mathieu P, Larose E, et al. Abdominal obesity and the metabolic syndrome: contribution to global cardiometabolic risk. ArteriosclerThromb Vasc Biol. 2008;28(6):1039-49.

25. Cameron AJ, Boyko EJ, Sicree RA, Zimmet PZ, Soderberg S, AIberti KG, et al. Central obesity as a precursor to the metabolic syndrome in the AusDiab Study and Mauritius. Obesity (Silver Spring). 2008. [Epub ahead of print].

26. Lin LY, Kuo HK, Li HY, Hwang JJ, Lin JW. Confirming a biological pathway in the metabolic syndrome-insight from the NHANES 1999-2002. Obesity (Silver Spring). 2008. [Epub ahead of print].

27. Reaven GM. Insulin resistance, the insulin resistance syndrome, and cardiovascular disease. Panminerva Med. 2005;47(4):201-10.

28. Shehata MF. Genetic and dietary salt contributors to insulin resistance in Dahl salt-sensitive (S) rats. Cardiovasc Diabetol. 2008;7:7.

29. Ruivo GF, Leandro SM, do Nascimento CA, Catanozi S, Rocha JC, Furukawa $L N$, et al. Insulin resistance due to chronic salt restriction is corrected by alpha and beta blockade and by L-arginine. Physiol Behav. 2006;88(4-5):364-70.

30. Donovan DS, Solomon CG, Seely EW, Williams GH, Simonson DC. Effect of sodium intake on insulin sensitivity. Am J Physiol. 1993;264(5 Pt 1):E730-4.

31. Foo M, Denver AE, Coppack SW, Yudkin JS. Effect of salt-loading on blood pressure, insulin sensitivity and limb blood flow in normal subjects. Clin Sci (Lond). 1998;95(2):157-64.

32. Raji A, Williams GH, Jeunemaitre $X$, Hopkins PN, Hunt SC, Hollenberg NK, et al. Insulin resistance in hypertensives: effect of salt sensitivity, renin status and sodium intake. J Hypertens. 2001;19(1):99-105.

33. Strazzullo P, Barbato A, Galletti F, Barba G, Siani A, lacone R, et al. Abnormalities of renal sodium handling in the metabolic syndrome. Results of the Olivetti Heart Study. J Hypertens. 2006;24(8):1633-9.

34. Sarafidis PA, Bakris GL. The antinatriuretic effect of insulin: an unappreciated mechanism for hypertension associated with insulin resistance? Am J Nephrol. 2007;27(1):44-54. 
35. Ohta Y, Tsuchihashi T, Arakawa K, Onaka U, Ueno M. Prevalence and lifestyle characteristics of hypertensive patients with metabolic syndrome followed at an outpatient clinic in Fukuoka, Japan. Hypertens Res. 2007;30(11):1077-82.

36. Hoffmann IS, Cubeddu LX. Salt and the metabolic syndrome. Nutr Metab Cardiovasc Dis. 2009;19(2):123-8.

37. Nakandakare ER, Charf AM, Santos FC, Nunes VS, Ortega K, Lottenberg AM, et al. Dietary salt restriction increases plasma lipoprotein and inflammatory marker concentrations in hypertensive patients. Atherosclerosis. 2008;200(2):410-6.

38. Jurgens G, Graudal NA. Effects of low sodium diet versus high sodium diet on blood pressure, renin, aldosterone, catecholamines, cholesterols, and triglyceride. Cochrane Database Syst Rev. 2004;(1):CD004022.

39. Lien LF, Brown AJ, Ard JD, Loria C, Erlinger TP, Feldstein AC, et al. Effects of PREMIER lifestyle modifications on participants with and without the metabolic syndrome. Hypertension. 2007;50(4):609-16.

40. Ard JD, Grambow SC, Liu D, Slentz CA, Kraus WE, Svetkey LP, et al. The effect of the PREMIER interventions on insulin sensitivity. Diabetes Care. 2004;27(2):340-7.

41. Townsend RR, Kapoor S, McFadden CB. Salt intake and insulin sensitivity in healthy human volunteers. Clin Sci (Lond). 2007;113(3):141-8.

42. Straznicky NE, Lambert EA, Lambert GW, Masuo K, Esler MD, Nestel PJ. Effects of dietary weight loss on sympathetic activity and cardiac risk factors associated with the metabolic syndrome. J Clin Endocrinol Metab. 2005;90(11):5998-6005.

43. Hoffmann IS, Cubeddu LX. Increased blood pressure reactivity to dietary salt in patients with the metabolic syndrome. J Hum Hypertens. 2007;21(6):438-44.
44. Uzu T, Kimura G, Yamauchi A, Kanasaki M, Isshiki K, Araki S, et al. Enhanced sodium sensitivity and disturbed circadian rhythm of blood pressure in essential hypertension. J Hypertens. 2006;24(8):1627-32.

45. Vedovato M, Lepore G, Coracina A, Dodesini AR, Jori E, Tiengo A, et al. Effect of sodium intake on blood pressure and albuminuria in Type 2 diabetic patients: the role of insulin resistance. Diabetologia. 2004;47(2):300-3.

46. Azadbakht L, Mirmiran P, Esmaillzadeh A, Azizi T, Azizi F. Beneficial effects of a Dietary Approaches to Stop Hypertension eating plan on features of the metabolic syndrome. Diabetes Care. 2005;28(12):2823-31.

47. GomiT, ShibuyaY, Sakurai J, Hirawa N, Hasegawa K, IkedaT. Strict dietary sodium reduction worsens insulin sensitivity by increasing sympathetic nervous activity in patients with primary hypertension. Am J Hypertens. 1998;11(9):1048-55.

48. He FJ, MacGregor GA. Effect of longer-term modest salt reduction on blood pressure. Cochrane Database Syst Rev. 2004;(3):CD004937.

49. Vogt TM, Appel LJ, Obarzanek E, Moore TJ, Vollmer WM, Svetkey $\mathrm{LP}$, et al. Dietary approaches to stop hypertension: rationale, design, and methods. DASH Collaborative Research Group. J Am Diet Assoc. 1999;99(8 Suppl):S12-8.

50. Van Horn L, McCoin M, Kris-Etherton PM, Burke F, Carson JA, Champagne $\mathrm{CM}$, et al. The evidence for dietary prevention and treatment of cardiovascular disease. J Am Diet Assoc. 2008;108(2):287-331.

51. Klein S. Outcome success in obesity. Obes Res. 2001;9 Suppl 4:354S-8S.

52. Wallace TM, Levy JC, Matthews DR. Use and abuse of HOMA modeling. Diabetes Care. 2004;27(6):1487-95. 